\title{
O CÂNCER DE MAMA NAS PÁGINAS DOS ANAIS BRASILEIROS DE GINECOLOGIA ${ }^{1}$
}

\author{
Breast Cancer in the Brazilian Annals of Gynecology` pages
}

\section{Vanessa Lana* Luiz Antonio da Silva Teixeira**}

\begin{abstract}
Resumo: O presente artigo analisa o percurso do pensamento médico na configuração do câncer de mama como doença e a organização de conhecimentos e práticas sobre a mesma no Brasil em meados do século XX, tendo como fonte os Anais Brasileiros de Ginecologia. O recorte temporal da análise corresponde ao início das discussões e ações em relação aos cânceres ginecológicos, na década de 1930, e a introdução do mamógrafo como instrumento de diagnóstico do câncer de mama no país, nos anos 1970, que impactou diretamente as práticas de controle da doença. O argumento principal é que a visão do campo médico expressa no periódico se apresenta de duas formas bastante diferenciadas, relacionadas ao processo de ampliação dos conhecimentos sobre os mecanismos da doença. Num primeiro momento, observa-se indefinições e busca de explicações e configurações sobre a doença. No segundo, como resultado de uma melhor identificação das formas de diagnóstico e possibilidades de intervenção e tratamento, busca-se a organização de ações de controle. Ao tomar os Anais Brasileiros de Ginecologia como principal fonte na construção desse trabalho, destacamos o papel que publicações especializadas têm na configuração de pensamentos e práticas, moldando saberes, agendas e perfis profissionais. Sendo a principal revista em ginecologia no período, os discursos ali trabalhados caminhavam no sentido de consolidação da própria ginecologia como especialidade médica.

Palavras Chave: Câncer de mama; Anais Brasileiros de Ginecologia; discurso médico.
\end{abstract}

\begin{abstract}
This article analyzes the path of medical thought in the configuration of breast cancer as a disease and the organization of knowledge and action practices in Brazil in the mid-twentieth century, having as source the Brazilian Annals of Gynecology. The time frame of the analysis corresponds to the beginning of discussions and actions regarding gynecological cancers in the 1930s, and the introduction of the mammograph as a breast cancer diagnostic tool in the country in the 1970s, which directly impacted control practices of the disease. The main argument is that the medical field view expressed in the journal presents itself in two very different ways, related to the process of expanding knowledge about the mechanisms of the disease. At first, there are

\footnotetext{
10 presente trabalho foi realizado com apoio do CNPq, Conselho Nacional de Desenvolvimento Científico e Tecnológico - Brasil (150214/2019-7). Apoio Fundação de Amparo à Pesquisa do Estado do Rio de Janeiro (Faperj) - Programas E-26/202.805/2018 - CNE. E-26/210.115/2016 - Humanidades.

* Doutora em História das Ciências - Casa de Oswaldo Cruz / FIOCRUZ. Pós doutorado (em curso) na Casa de Oswaldo Cruz / FIOCRUZ. Professora do Departamento de História da Universidade Federal de Viçosa. Professora do Mestrado Profissional em Patrimônio Cultural, Paisagens e Cidadania - UFV. ORCID: http://orcid.org/0000-0002-1950-1206 E-mail: vanlana@uol.com.br

** Doutor em História Social. Pesquisador no Departamento de Pesquisa e no Programa de Pós-graduação em História das Ciências e da Saúde, da Casa de Oswaldo Cruz / FIOCRUZ. Professor no Programa de Pósgraduação em Saúde da Criança e da Mulher, do Instituto Fernandes Figueira. Pesquisador de produtividade pelo CNPq. Cientista do Nosso Estado pela Faperj. E-mail: luiztei3@gmail.com
} 
undefinedness and search for explanations and configurations about the disease itself. In the second, as a result of a better identification of the forms of diagnosis and possibilities of intervention and treatment, the organization of control actions is sought. Taking the Brazilian Annals of Gynecology as the main source in the construction of this work, we highlight the role that specialized publications have in shaping thoughts and practices, shaping knowledge, agendas and professional profiles. Being the main journal in gynecology in the period, the speeches worked there aimed at consolidating gynecology itself as a medical specialty.

Key Words: Breast Cancer; Brazilian Annals of Gynecology; medical discource.

\section{Introdução}

O objetivo desse artigo é analisar o discurso médico sobre o câncer de mama em meados do século XX no Brasil, tendo como fonte os Anais Brasileiros de Ginecologia, um dos principais veículos de circulação do conhecimento científico sobre ginecologia no período. O contexto de análise, entre as décadas de 1930 e 1970, reflete, respectivamente, o início das discussões e ações em relação aos cânceres ginecológicos, e a introdução do mamógrafo como instrumento de diagnóstico do câncer de mama no país, o que impactou diretamente as práticas de controle da doença.

O argumento principal é que a visão do campo médico expressa nos Anais Brasileiros de Ginecologia se apresenta de duas formas bastante diferenciadas, relacionadas ao processo de ampliação dos conhecimentos sobre os mecanismos da doença. Num primeiro momento, observa-se indefinições e busca de explicações e configurações sobre a doença em si. No segundo momento, como resultado de uma melhor identificação das formas de diagnóstico e possibilidades de intervenção e tratamento, busca-se a organização de ações de controle. Partindo dessa perspectiva, o artigo discute o percurso do pensamento médico na configuração do câncer de mama como doença e a organização de conhecimentos e práticas de ação sobre a mesma.

Ginecologia e obstetrícia, até o final do século XIX, caminhavam em conjunto no tocante aos cuidados com a mulher. Ambas surgiram vinculadas ao ideal de que a reprodução seria o principal objetivo da vida feminina, e por conta disso um determinante de seus aspectos fisiológicos (MOSCUCCI, 1990). A constituição da ginecologia como campo de conhecimentos médicos esteve relacionada ao desenvolvimento de práticas cirúrgicas na região abdominal feminina, como a histerectomia (extração do útero) e ovariotomia (extração dos ovários), a embriotomia (extração do feto, na inviabilidade do nascimento) e o aperfeiçoamento das cesarianas. Tais técnicas se desenvolveram ao longo do século XIX, concomitantemente a inovações nos instrumentos cirúrgicos, que possibilitaram a ampliação das intervenções médicas no corpo feminino (FREITAS, 2008; MOSCUCCI, 1990).

No último quartel do XIX, ginecologia e obstetrícia se separaram, como especificidades médicas, atentando cada qual para suas finalidades principais. Enquanto os obstetras tinham de se sobrepor às parteiras na assistência ao parto, atraindo a confiabilidade feminina, os ginecologistas buscavam vencer o pudor feminino frente à exposição de seus corpos, para que estas aceitassem se submeter a um exame ginecológico. Tal estratégia era fundamental para a medicalização dos corpos femininos e consequente ampliação do rol de ações da medicina (MARTINS, 2004).

No Brasil, o surgimento das especialidades médicas voltadas para a mulher acompanhou a organização das escolas médico-cirúrgicas no início do século XIX. Em 1809, um ano após a criação das escolas em Salvador e no Rio de Janeiro, a cadeira de partos, denominada "Moléstias das mulheres pejadas e dos meninos recém-nascidos" era parte integrante do currículo (FREITAS, 
2008). De forma semelhante à Europa, no Brasil, a medicina da mulher neste período era essencialmente cirúrgica e relacionada à atividade de partos. No final do século XIX foi criada a cátedra de Clínica Obstétrica e Ginecologia e, em 1884, as faculdades de medicina brasileiras passaram a oferecer, em dois anos, o curso de Obstetrícia e Ginecologia (IDEM).

A Ginecologia brasileira ganhou espaço a partir do início do século XX. A "ciência da mulher" era valorizada por sua função de garantir a procriação e o bom encaminhamento da maternidade, considerada papel primordial da mulher. Naquele momento, ao caráter preponderantemente teórico da disciplina, foi incorporada a prática profissional, principalmente com o surgimento de hospitais para o exercício da especialidade. O período foi caracterizado por uma intensificação no intercâmbio de ideias, com a organização de instituições especializadas, como associações, congressos e publicações na área. Assim, o processo de medicalização do corpo feminino no Brasil teve seu início com a construção de espaços hospitalares específicos para atendimento das especialidades, fornecendo as condições necessárias para o ensino da clínica e pesquisas científicas (MARTINS, 2004).

Os anos de 1930 foram marcados por mudanças importantes no cenário da ginecologia brasileira. Até aquele momento, o ensino da especialidade era realizado em conjunto com a cirurgia geral, tendo uma roupagem mais teórica na formação profissional. Tal cenário se transformaria a partir de 1936, quando foi criada uma cátedra específica de ginecologia - desvinculada da cirurgia - na Faculdade de Medicina da Universidade do Brasil, no Rio de Janeiro. Nesse momento, o médico Arnaldo de Moraes (1893 - 1961) tornou-se o catedrático da nova disciplina. Seu nome e sua trajetória profissional estão diretamente relacionados à história do desenvolvimento da ginecologia no Brasil. A partir de 1936, ele reorganizou a Clínica Ginecológica da Universidade e passou a coordenar suas atividades. Em 1947 o fortalecimento da pesquisa no setor possibilitou sua transformação em Instituto de Ginecologia, e sua transferência para instalações no Hospital Moncorvo Filho (LANA, 2016).

Ainda em 1936, Arnaldo fundou o periódico Anais Brasileiros de Ginecologia, caracterizado como órgão oficial do ensino de ginecologia na Universidade (Anais Brasileiros de Ginecologia editorial, vol, 1, n. 1, 1936). Periódico mensal dedicado à "ciência da mulher", os Anais se tornaram um espaço institucional importante para o fortalecimento do campo da ginecologia. A revista era uma publicação mensal e, segundo seus organizadores, de cunho exclusivamente científico. Dedicado ao ensino da "ciência da mulher", o periódico, segundo seu editor, buscava ser capaz de "honrar a ciência médica brasileira e de [dialogar] com as congêneres estrangeiras". Na primeira edição, a apresentação o classificava como espaço de fomento às discussões médicas e espaço de construção de conhecimentos científicos (LANA, 2012).

A publicação veiculou mensalmente, de forma ininterrupta, até o ano de 1970, quando, mantendo o corpo profissional e de colaboradores, mudou de denominação para Jornal Brasileiro de Ginecologia (LANA, 2012). Segundo Bonan e colaboradores, os Anais Brasileiros de Ginecologia foram fruto do processo de renovação da ginecologia brasileira, ocorrido em meados dos anos 1930 e tiveram em sua base uma autoimagem de agentes de modernização da medicina nacional e busca de sua internacionalização (BONAN, TEIXEIRA E NAKANO, 2017). Por seu alcance e importância no campo profissional, Os Anais são uma importante referência para se compreender o discurso médico sobre o controle do câncer ginecológico no Brasil do século XX. Assim, tomamos a publicação como principal fonte de pesquisa nesse artigo, analisando a configuração social e os aspectos científicos do câncer de mama discutidos no Brasil entre as décadas de 1930 e 1970.

Entre os anos de 1937, quando começaram a existir, e os anos 1970, quando sob nova 
configuração a revista passa a ser denominada Jornal Brasileiro de Ginecologia, foram publicados 38 artigos sobre câncer de mama. Esse número se mostra bastante baixo frente ao quantitativo de artigos publicados pelo periódico nesse mesmo período. No entanto, sua distribuição mostra-se constante, com a ampliação do número de artigos ao final do período, consoante ao desenvolvimento da preocupação com a doença desenvolvida na sociedade, em especial, a partir do surgimento de técnicas mais eficazes para o seu tratamento ${ }^{2}$.

\section{Discutindo a doença e a detecção precoce}

Nesse trabalho, entendemos uma doença não como algo dado e determinado a priori, mas sim como um fenômeno biológico e social, que carrega consigo significados reveladores de uma determinada sociedade (ROSENBERG, 1997). A doença, neste sentido, adquire significados mais complexos do que os da dimensão simplesmente biológica. Em outras palavras, encerra em si questões sociais, culturais, políticas e econômicas. É parte integrante de um jogo de interesses, de formação e construção da identidade da doença pelo corpo profissional e da incidência desta no meio social (SILVEIRA E NASCIMENTO, 2004).

A existência de uma doença provem de um acordo socialmente dado entre grupos sociais: médicos, cientistas, pacientes, famílias. É possível reconheça-la quando esta, em algum momento, torna-se uma questão médica e, para tal, é necessário, na mesma medida, o próprio reconhecimento e aceitação social da categoria que se está "enquadrando". O fenômeno doença é delineado socialmente como entidade específica, que se constitui e se manifesta mediante características particulares quando há acordo sobre ela. O enquadramento de uma doença reflete e incorpora valores e atitudes inseridos em um contexto cultural. Neste sentido, são necessárias não apenas a identificação, mas também, a aceitação social, entendendo-a como fruto de um acordo coletivamente produzido (ROSENBERG, 1997).

Assim, podemos argumentar que as discussões sobre o câncer de mama, no caso específico do Brasil, remetiam a uma necessidade social de criação e formulação de uma explicação sobre a enfermidade em si. Num momento de consolidação da própria ginecologia como especialidade médica, os crescentes índices de problemas ginecológicos em mulheres demandavam, por parte destes profissionais, respostas e medidas de conhecimento e controle do problema. Tal demanda partia da própria atividade profissional exercida e da sociedade, acometida com uma enfermidade ainda muito misteriosa e sobre a qual giravam inúmeras especulações e crendices. Neste sentido, um diagnóstico mais preciso e formas de controle mais eficazes, propiciavam ao grupo médico maior autoridade nos discursos e na formação de políticas de intervenção no corpo doente.

A ideia de "enquadramento" nos permite pensar na formação dos conhecimentos e práticas sobre o câncer de mama, discutidos e organizados pelos ginecologistas brasileiros em meados do século XX. Pensar, portanto, na construção de um conhecimento específico e de um corpo profissional para controle da enfermidade a partir dos referenciais do enquadramento da doença, acima apresentados, nos permite problematizar os discursos que envolveram a tomada do câncer de mama como questão de saúde, e da própria construção de um discurso sobre o mesmo. E, na mesma medida, na construção de uma imagem médica em torno doença e da formação de uma política conjunta, articulada nacionalmente.

\footnotetext{
2 Artigos tematizando o câncer de mama nos Anais Brasileiros de Ginecologia por ano: 1937-1; 1938- 2; 1939-1; 1940-1; 1941-1;1942-1;1945-1; 1946-4;1949-1;1951-1;1952-2;1955-1;1956-3; 1957-1;1964$1 ; 1965-1 ; 1966-3 ; 1968-1 ; 1969-1970-11$
} 
A demanda por explicações que abarcassem as multiplicidades da doença canalizaram esforços para a construção de discursos sobre a enfermidade em si e, mais propriamente, das formas de controle e prognóstico do tratamento. Um dos pontos principais da elaboração dos discursos versou sobre a necessidade de diagnóstico precoce dos tumores, e da busca por cuidados médicos mesmo sem a presença de sinais indicativos da doença.

A ideia de diagnóstico precoce e prevenção veiculada às ações de controle do câncer de mama de meados do século XX estava relacionada às discussões trazidas pelo chamado movimento da medicina preventiva, originado no período de 1920 a 1950, como forma de crítica à medicina curativa. Inicialmente pensado e proposto em Inglaterra, EUA e Canadá, esse movimento propôs uma mudança na prática médica por intermédio de reformas no ensino, buscando destacar a responsabilidade dos profissionais em promover saúde e prevenir doenças.

A base conceitual deste movimento foi sistematizada por Leavell e Clark, em 1965, num que estudo busca relacionar as diversas fases do adoecimento à dimensões sociais e ambientais. $\mathrm{O}$ modelo por eles desenvolvido foi uma tentativa de esquematizar a organização da intervenção humana sobre o processo saúde-doença a partir da organização dos conceitos de promoção, prevenção, cura e reabilitação, em um modelo denominado "História Natural da Doença" (LEAVELL E CLARK, 1976). Na abordagem de Leavell e Clark, adoecimento e vida saudável não dependem unicamente de aspectos físicos e/ou genéticos. As condições de saúde estariam relacionadas a fatores específicos como: o patrimônio biológico, as condições sociais e econômicas no ambiente, estilo de vida, acesso à alimentação, educação, trabalho, renda, lazer e intervenções de ordem médica (NUNES, 1974).

Para a discussão aqui realizada, é importante compreender que o desenvolvimento do conceito de história natural da doença e das noções a ele associadas, possibilitaram a ampliação e maior definição das ideias sobre prevenção e diagnóstico precoce, sendo que ambos muitas vezes foram vistos como sinônimos nas ações de controle do câncer. Até meados do século XX, prevenir a doença correspondia a identificar seus possíveis sinais, mesmo antes de sua manifestação física. A realização do diagnóstico precoce, nesse sentido, era tomada como principal estratégia de prevenção.

Tomando o diagnóstico precoce como principal estratégia de prevenção, a mensagem das campanhas de controle da doença era a de que a cirurgia, primeiro método de tratamento utilizado, seria mais eficaz quanto menos os pacientes retardassem a procurar os cuidados médicos aos primeiros sinais ou suspeita da enfermidade. Tal mensagem aludia à responsabilidade individual do paciente para recorrer ao tratamento médico e garantir possibilidade de sucesso na terapêutica e melhor prognóstico (ARONOWITZ, 2001).

O discurso que relacionava a rapidez na busca por cuidados médicos e a o aumento das possibilidades de êxito com a cirurgia foi o argumento de força ressaltado pelas campanhas de saúde, focadas na redução da demora das pacientes em buscar atendimento médico, tanto no Brasil quanto em outros espaços como Europa e EUA. O tempo exercia um papel complementar na cirurgia extensiva, criando uma rede de responsabilidade em câncer, uma responsabilidade negociada entre pacientes e médicos, para obtenção de resultados satisfatórios (ARONOWITZ, 2001).

As mudanças nas abordagens sobre o câncer de mama estiveram condicionadas às negociações de diferentes atores e grupos sociais em torno das técnicas de detecção precoce. Na primeira metade do século, a autoridade dos cirurgiões quanto à prática da mastectomia delimitou não somente um espaço de atuação, mas também um universo de relações entre os médicos especialistas, a saúde pública e as mulheres doentes, que era dominado pelos cirurgiões. Principalmente a partir 
dos anos 1970, porém, a entrada em jogo de um novo conjunto de conhecimentos - vinculado à medicina de risco, à epidemiologia e à biomedicina - e de tecnologias, principalmente as de visualização da mama, reorientou o eixo de discussões sobre a doença para a prevenção. Articulados às demandas dos movimentos de mulheres, esses saberes e técnicas possibilitaram a formulação de discussões específicas sobre câncer e saúde feminina.

Apesar da aceitação do campo médico, a violência da cirurgia e a pequena taxa de sobrevida impedia um olhar mais otimista para o procedimento. Como indicado por diversos autores, na impossibilidade de uma terapêutica eficaz, o câncer de mama, tal qual outros cânceres, teriam na ideia de prevenção e detecção precoce sua forma principal de controle (CANTOR, 2008). Como predominava a concepção de que o câncer era proveniente de processos inflamatórios e lesões originárias de choques mecânicos, os médicos propunham a constante vigilância em relação a manchas, sangramentos, protuberâncias e outras "anomalias" nos seios (TEIXEIRA e ARAUJO NETTO, 2020). Esse modelo de atenção constante, também comum em outros países, Inglaterra e França, se tornaria a principal forma de controlar a doença (ARONOWITZ, 2001).

\section{Combatendo o câncer de mama}

As primeiras discussões em relação ao câncer de mama se deram no âmbito da clínica, tendo como centro a cirurgia (TEIXEIRA E ARAÚJO NETO, 2020). No início do século XX, o câncer de mama era visto como uma doença de mulheres idosas e, embora não houvesse explicação para a causa, ela era vista como incurável, sendo tratada somente a partir da cirurgia, no caso a mastectomia radical, técnica desenvolvida pelo cirurgião estadunidense William Halsted, no final do século XIX (CONTI, 1912).

A cirurgia de Halsted, embora extremamente agressiva, foi a principal forma de tratamento, por fornecer melhores perspectivas do que qualquer outro procedimento tentado até então (OLSON, 2002). No entanto, um número pequeno de mulheres tinha acesso a esse tipo de cuidado, ou mesmo tinham interesse em se submeter a uma intervenção que lhes ressecaria os principais músculos do tórax (externo e peitoral), muitas vezes também retirando seu braço. Apesar de pouco utilizada, a mastectomia radical foi alvo de muitas discussões médicas no início do século XX, principalmente a partir de 1920. Artigos em periódicos especializados (Brazil Médico, Revista Brasileira de Medicina, Archivos Brasileiros de Medicina) e teses da faculdade de Medicina do Rio de Janeiro discutiram os seus resultados, indicando a possibilidade de sobrevida das mulheres entre 3 e 5 anos, nos melhores cenários; um avanço considerável aos olhos dos cirurgiões no período (SANTOS JÚNIOR, 1923).

No final dos anos 1930, quando o câncer ganhou mais visibilidade, a partir das iniciativas do cirurgião Mario Kroeff - que deu início ao tratamento da doença por eletrocirurgias e fundou o Centro de Cancerologia no Rio de Janeiro - a utilização da eletrocirurgia nas mastectomias radicais passou a ser postulada pelos mais influentes cirurgiões relacionados ao câncer ginecológico. Dollinger da Graça, professor da Faculdade de Medicina do Rio de Janeiro e um dos mais reputados cirurgiões da cidade, Ugo pinheiro Guimarães e Alberto Coutinho importantes nomes do centro de cancerologia e mais tarde do Serviço Nacional de Câncer, seriam defensores da utilização dessa técnica como forma de diminuir o sangramento e facilitar incisões mais amplas (KROEFF, 2007).

Nos anos 1940, quando a ampliação da medicina previdenciária ensejou a criação de diversos hospitais no país, as cirurgias de câncer de mama passam a ter maior disseminação. Naquele 
momento, novas técnicas de controle da doença começaram a ser empregadas junto a cirurgia radical. A busca de formas específicas de exames de radiográficos que mostrassem com maior precisão tumores imperceptíveis a apalpação, maior uso da radioterapia prévia para diminuir o tamanho do tumor e a radioterapia pós-operatória passaram a marcar o que os médicos do período chamavam de "arsenal de armas contra o câncer" (COUTINHO, 1941; GUIMARÃES, 1942).

No campo da saúde pública, as ações relacionadas à doença tiveram por base a campanhas de esclarecimento quanto à necessidade de diagnóstico precoce. Em relação a esse ponto, a criação do Serviço Nacional de Câncer, em 1942, ampliou a noção de educação em saúde como melhor forma de controle. Em relação ao câncer de mama, como aos outros tipos da doença o modelo de prevenção tinha como base a noção que passou para a história como cancerofobia, ou seja, a possibilidade de causar medo em relação à doença para que a pessoa procurasse um especialista ao primeiro sinal de anormalidade. Exposições com modelos de gesso ou fotos de doentes - expondo as partes atingidas de forma trágica - e cartazes explicando os tipos da doença de forma assustadora foram a marca da educação em saúde sobre o câncer em meados do século XX (TEIXEIRA E GRUZMAN, 2017).

Nesse mesmo período, o país passava por um processo de centralização de serviços de saúde que determinou, em 1943, a criação do Serviço Nacional de Câncer, setor da saúde pública responsável pela coordenação de ações contra a doença - tanto as públicas como as de origem filantrópicas - e pela organização da Campanha Nacional Contra o Câncer, modelo de ação que buscava juntar esforços públicos, privados e filantrópicos para combater a enfermidade. A partir desses setores, as ações educação sanitária e os primeiros hospitais especializados na doença começaram a se disseminar pelo país (TEIXEIRA E FONSECA, 2007).

O desenvolvimento dos conhecimentos e práticas dirigidas aos cânceres femininos, nesse período, teve como base a criação e desenvolvimento de algumas instituições especializadas. $\mathrm{O}$ Instituto de Ginecologia da Faculdade de Medicina do Rio de Janeiro, o Hospital de Ginecologia em Minas Gerais e o Hospital Aristides Maltez na Bahia foram as bases de organização do campo da cancerologia voltada para a saúde das mulheres e, em especial para o câncer de mama (LANA, 2012). Em relação à organização do campo profissional, o surgimento da Sociedade Brasileira de Patologia Mamária, em 1959 foi um importante marco. A partir da década de 1970, renomeada de Sociedade Brasileira de Mastologia, a sociedade criou regionais em diversos Estados do país, nas quais cirurgiões e ginecologistas recebiam formação continuada e estimulavam ações públicas e privadas para o controle do câncer de mama no país. A Sociedade Brasileira de Mastologia teve grande contribuição na elaboração de políticas e normas direcionadas ao câncer de mama (PORTO; TEIXEIRA; SILVA, 2013).

A partir da década de 1960, o desenvolvimento de novas tecnologias de diagnóstico transformou o cenário do câncer de mama no país. Em relação ao diagnóstico, a confirmação epidemiológica da percepção de que a identificação de pequenas alterações nos seios, somente observáveis por meio radiológico, poderia gerar intervenções mais eficazes para a cura da doença fez com que se ampliasse a busca por tecnologias de visualização das estruturas da mama. Desde a década de 1940, a radiologia era bastante utilizada no diagnóstico em âmbito da medicina individual. A partir dos anos 1960, o desenvolvimento de novas técnicas diagnósticas de visualização do interior das mamas, como o transiluminação e a mastografia, reforçaram a preocupação com o diagnóstico precoce (GÓES JÚNIOR, 1967). Mas seria o desenvolvimento do mamógrafo, no início dos anos 1970, que tornaria viável o objetivo de ampliar o diagnóstico precoce da doença em nível em nível populacional. A partir desse período, algumas instituições públicas ou filantrópicas - como as 
Pioneiras Sociais - e diversos consultórios privados começaram a adquirir mamógrafos e treinar seus médicos para o diagnóstico precoce de tumores mamários, dando início a uma forma de pensar o controle do câncer baseada no conhecimento precoce da doença a partir do exame mamográfico de diagnóstico ou de rastreamento.

\section{Os discursos sobre o câncer de mama nos Anais Brasileiros de Ginecologia}

Os primeiros artigos sobre câncer de mama publicados nos Anais Brasileiros de Ginecologia datam dos anos 1930, logo quando da criação da revista, discutindo as definições sobre a doença, quadros de diagnóstico e procedimentos a serem adotados para identificação e tratamento. O editorial, de 1939, intitulado "carcinoma da mama", destacava as imprecisões na definição da doença, do tratamento, e no conhecimento de suas causas. O grande debate naquele momento girava em torno da identificação do tumor. Num momento anterior a difusão da mamografia como tecnologia de diagnóstico, a detecção precoce se dava com o exame clínico das mamas, exames de raio X e biópsia. Segundo o editorial: "a mama que sangra, acompanhada de tumoração, não apresenta dificuldades ao diagnóstico, mas se esta última não existe, o problema é mais difícil”" (Anais Brasileiros de Ginecologia - Editorial, vol. 7, n. 3, 1939).

A detecção precoce era, assim a principal bandeira já levantada pelos médicos, tendo como preceito que um prognóstico positivo de câncer de mama se daria com a identificação e extirpação rápidas do tumor. Para possibilitar a realização da detecção do tumor em fases iniciais, o discurso era da defesa do exame sistemático e rotineiro das mamas em todos os exames ginecológicos. E para além do exame, preconizava-se a realização de biópsia em todos os tumores clinicamente suspeitos de malignidade, e indicação para operação em até $48 \mathrm{~h}$ de todos os casos confirmados com o exame (ZANOTTA, 1955).

A identificação precoce do carcinoma da mama tomou um papel importante nesse contexto, pela função que representava na própria configuração social da doença. Isso porque, o ato do diagnóstico estrutura a prática médica, confere aprovação social dos papeis da doença e legitima as relações burocráticas. Segundo Charles Rosenberg, a realização do diagnóstico exerce um papel central na definição do fenômeno social chamado doença. É o ponto de articulação entre o médico e o paciente, sendo o mecanismo de comunicação e de identidade (ROSENBERG, 2002). Através do diagnóstico, a doença é especificada e ocorre um processo de legitimação tanto da prática médica quanto do papel de uma determinada enfermidade no meio social.

A história moderna do diagnóstico está relacionada à especificidade da doença. Durante o século XX, as categorias de diagnóstico, prognóstico e tratamento estiveram ligadas cada vez mais rigorosamente a categorias específicas de doenças, no conceito e na prática cotidiana. O sistema de categorias e diagnóstico é tanto uma metáfora para nossa sociedade quanto um microcosmo da mesma. Nesse sentido, Rosenberg argumenta que "o diagnóstico é um elemento substantivo desse sistema, uma chave para o repertório de senhas que fornecem acesso ao software institucional que gerencia a medicina contemporânea. Isso ajuda a tornar a experiência da máquina legível” (ROSENBERG, 2002, p. 257). As técnicas de diagnóstico produzem diferentes formas de se pensar sobre uma enfermidade, e possibilitam a própria construção da categoria em questão.

A definição do diagnóstico do câncer de mama caminhava em paralelo à própria definição das tipologias do tumor. Em artigo publicado em 1946, o médico Aurélio Monteiro, docente da Faculdade de Medicina da Universidade do Brasil no Rio de Janeiro, mostrava as dificuldades, no atual estado da oncologia, em estabelecer com precisão todas as variedades de neoplasias epiteliais 
malignas (MONTEIRO, 1946). Como qualquer campo científico, a observação de um novo problema implicava na criação de consensos sobre essa especialidade para a criação de protocolos.

$\mathrm{Na}$ discussão sobre o diagnóstico precoce da doença, como estratégia de prevenção e de melhoria no seu prognóstico, o médico Alberto Coutinho, do Serviço Nacional do Câncer, publicou um artigo intitulado "Radiodiagnose da mama", discutindo os Raios X como ferramenta de detecção do câncer de mama. Na publicação, o método fora considerado de valor secundário em comparação ao exame clínico e anátomo-patológico das mamas. Isto porque, as imagens em raios$\mathrm{X}$ apresentavam modificações em casos de gravidez, menstruação, lactação e menopausa (COUTINHO, 1946).

O artigo de Coutinho é interessante para discutir as definições em torno da doença e seu diagnóstico. O médico descreveu os registros do primeiro ano de atividades do Centro de Cancerologia no Rio de Janeiro, versando sobre câncer de mama e afecções correlatas. Trouxe um quadro do perfil das pacientes diagnosticadas com a doença, todas mulheres, a maioria na faixa de 50 e 60 anos. As pacientes atendidas teriam notado o tumor de forma acidental. A dor não foi causalidade presente na identificação do tumor pelas pacientes, isso porque a dor geralmente ocorria em casos ulcerados (IDEM).

Discorrendo sobre as definições da doença e dos procedimentos a serem adotados, o médico destacava que a propagação do câncer mamário se daria majoritariamente por via linfática. Assim, um dos maiores indicadores do grau de invasão seria o comprometimento dos vasos e gânglios desse sistema. O diagnóstico seria otimizado pela realização de biópsias em todos os casos com tumores identificados, com menos tempo possível entre o exame histopatológico e a cirurgia, para evitar metástases nesse intervalo, pois o foco canceroso poderia permanecer aberto facilitando a migração de células blastomatosas. O prognóstico da doença, nesse sentido, era pensado a partir da conjunção de três fatores: as condições clínicas da paciente, o tipo histológico do tumor e o tratamento (COUTINHO, 1946).

Em relação ao tratamento, era consensual nos artigos a defesa da mastectomia radical, ou cirurgia de Hasteald. A mastectomia consiste na exérese, ou retirada, da glândula mamária. Há dois tipos principais: a mastectomia simples (que compreende a retirada de toda a mama), e a mastectomia radical, ou operação de Halsted (lançada em 1882), que compreende a retirada, em monobloco, da mama, de todo tecido gorduroso da região, dos músculos peitorais e linfonódios axilares (GÓES JR, 1970, p. 209).

No editorial dos Anais Brasileiros de Ginecologia, de 1939, a cirurgia era destacada como ocupante de um lugar proeminente no tratamento do carcinoma da mama (Anais Brasileiros de Ginecologia - Editorial, vol. 7, n. 3, 1939). Nesse mesmo sentido, Arnaldo de Moraes, discutindo a terapêutica cirúrgica da doença, argumentava que o trabalho do médico giraria em torno da identificação de duas categorias dos tumores mamários: operável e inoperável - por curável cirurgicamente e incurável cirurgicamente. O médico defendia que todas as mulheres em condições de ser operadas deveriam ser submetidas à mastectomia. Assim, "O tratamento por excelência do carcinoma da mama é a prática da operação radical em todos os casos presumidamente operáveis" (MORAES, 1949, p. 115).

O grupo ligado ao Centro de Cancerologia, sob direção de Mário Kroeff, defendia o uso da eletrocirurgia nas cirurgias mamárias. A cirurgia de Halsted (mastectomia radical), era utilizada no Centro, com algumas modificações na sequência dos tempos e com o uso da eletrocirurgia (VIANNA, 1940). Tal prática, décadas mais tarde, seria contestada pela comunidade médica, em termos de sua efetividade na terapêutica do câncer, como apresentaremos mais adiante. 
Ainda no campo da terapêutica, métodos auxiliares eram destacados como potenciais no combate à doença. A radioterapia, por exemplo, associada à cirurgia, melhoraria o prognóstico, aumentando a sobrevida. A prática de irradiacão era destacada em dois momentos no tratamento: pré e pós cirurgia. Como método pré-operatório, era proposta para diminuir os tumores existentes. Já as irradiações pós-operatórias, eram apontadas como fatores indiscutíveis no tratamento do câncer mamário, com vistas a destruir os elementos celulares neoplásicos que persistissem após a extirpação total da mama (COUTINHO, 1941). A irradiação pos-operatoria era, assim, defendida como medida profilática para impedir recidiva local, e como forma de melhorar a percentagem de cura. "quando se pratica uma verdadeira operação radical num carcinoma operável da mama, a doente não morre de recidiva local, mas de metástases a distância" (Anais Brasileiros de Ginecologia - Editorial, vol. 7, n. 3, p. 228, 1939). Uma frase que sistematiza o preceito da associação entre irradiação e cirurgia naquele momento era: "operar como se não fosse irradiar. E irradiar como se não tivesse operado" (VIANNA, 1940, p. 390). Ou seja, o discurso era de que ambas as práticas deveriam ser utilizadas de forma indiscutível, e, nos casos necessários, deveria se fazer o uso de ambas com vistas a obter melhores resultados no tratamento.

Outra discussão que esteve em cena nos anos 1930 e 1940, versando sobre formas de terapêutica e controle do carcinoma da mama, foi a castração radiológica nos casos inoperáveis ou com metástases, quando a doente ainda não tivesse atingido a menopausa, e adotando conduta semelhante em casos selecionados após a menopausa (Anais Brasileiros de Ginecologia - Editorial, 1939). A discussão sobre a castração radiologia nas mulheres operadas por câncer de mama era destacada principalmente pelo argumento da ação cancerígena dos hormônios estrogênicos (MORAES, 1949). Embora a real vinculação do estrogênio ao câncer de mama só tenha ocorrido no final da década de 1960, a descoberta do hormônio estrogênio quarenta anos antes, e a observação da relação desse hormônio com o câncer de próstata, observada pelo médico americano Charles Hugginsem, em 1941, permitiu o surgimento de hipóteses nesse sentido (OLSON, 2002). Entre os anos 1920 e 1940, uma verdadeira corrida em busca do desenvolvimento de hormônios estava ocorrendo nos EUA e Europa, o que ampliou as buscas da compreensão na fisiologia das mulheres e também seu papel em diferentes tipos de câncer (OUDSHOORN, 1994). Nesse contexto, o estrogênio passou a ser visto como um possível inibidor de cânceres de mama. Vários estudos, em diversas partes do mundo procuraram compreender a relação entre o câncer de mama e os ovários, discussão presente nos Anais Brasileiros de Ginecologia.

A partir dos pontos aqui levantados sobre as definições e questões acerca do diagnóstico e tratamento do câncer de mama no alvorecer das ações sobre a doença no Brasil, outras considerações sobre o estado das discussões podem ser apreendidas por um levantamento publicado no editorial dos Anais Brasileiros de Ginecologia, em 1952. O editorial sistematizava algumas conclusões retiradas de um simpósio sobre terapêutica do câncer de mama, realizado pela Sociedade Brasileira de Ginecologia. Segundo o material, o câncer de mama seria um dos mais complexos problemas clínicos com os quais se depararia um ginecologista. Isso porque, mesmo sendo o de maior incidência, a doença ainda não apresentava progresso satisfatório em relação a terapêutica. Mesmo com as cirurgias se tornando mais radicais, associadas a outros métodos de tratamento como a curieterapia e a roentgenterapia, os resultados finais mostravam poucas diferenças em seu percurso (Anais Brasileiros de Ginecologia - Editorial, vol 34, n. 1, 1952).

Haveria um certo consenso entre os profissionais da área de que a cura absoluta da doença não ultrapassaria 30\% dos casos diagnosticados. Para os casos operáveis, a mastectomia radical seria a melhor indicação terapêutica. Já nos casos inoperáveis, e em estados iniciais de evolução do 
tumor, as irradiações (com radioterapia) também seriam métodos indicados, com possibilidades de cura da paciente. As irradiações também seriam indicadas em casos de metástases ou recidivas, tanto com valor curativo (em poucos casos), quanto com valor paliativo, na grande maioria dos casos, prologando a sobrevida da paciente. Os resultados finais do tratamento, associando irradiações à operação radical, poderiam ser melhores que os obtidos somente com a terapêutica cirúrgica isolada (Anais Brasileiros de Ginecologia - Editorial, vol 34, n. 1, 1952).

Os pontos acima observados representam o estado da discussão sobre o câncer de mama na medicina brasileira da primeira metade do século XX, permanecendo sem maiores alterações até fins dos anos 1960. Os anos 1960 e início dos anos 1970, que antecedem a introdução do mamógrafo no Brasil como ferramenta de diagnóstico da doença, representam um endossamento da discussão até então realizada. Naquele contexto, a identificação, ou "enquadramento" da doença, enquanto categoria social e biológica, estava mais estruturada, o que abriu espaço para o aprofundamento de algumas questões e indagação a outros pontos sobre o carcinoma da mama. A realização do diagnóstico, da identificação e classificação das doenças é segundo Ilana Lowy, princípio fundamental da organização da medicina moderna (LOWY, 2010).

A busca por uma definição do câncer de mama e de seus estados clínicos, no estabelecimento de um consenso no diagnóstico, esteve presente ao longo da primeira metade do século XX. Em julho de 1954 foi realizado em São Paulo o VI Congresso Internacional do Câncer, no qual foi criado um Comitê para a Classificação dos Cânceres por Estágios Clínicos e seu Uso Estatístico. A proposta do comitê era da elaboração de um projeto para classificação clínica dos carcinomas mamários. Em 26 de outubro de 1956, a Sociedade Brasileira de Ginecologia votou a “Classificação Clínica dos Tumores Malignos da Mama” (VIVEIROS E ZANOTTA, 1956).

Com uma definição mais clara do que seria o carcinoma da mama, seus estriamentos clínicos, formas de diagnóstico e terapêutica, as discussões dos anos 1960 caminharam no sentido de endossamento, críticas e revisões às práticas até então empregadas. As publicações do início dos anos 1970, quando o periódico passa a ser denominado Jornal Brasileiro de Ginecologia, reservaram grande espaço para a temática do câncer de mama em seus diferentes aspectos.

É possível observar nas publicações desse período uma definição mais clara e consensual sobre a doença, considerando o câncer não como algo unitário, mas sim como um conjunto de entidades patológicas, que levam em consideração os agentes carcinogenéticos e o comportamento biológico dos tumores. Nesse sentido, os artigos trazem nesse período considerações mais específicas em relação ao tratamento, com indicação dos agentes terapêuticos para tipos específicos de tumores mamários femininos. Num momento em que as definições ou o processo de "enquadramento" da doença já havia se constituído, outras questões vinham à tona, como um aprofundamento nas discussões de práticas já adotadas em relação ao diagnóstico e, principalmente, ao tratamento do carcinoma mamário.

A quimioterapia, por exemplo, como método adjuvante à cirurgia foi um dos principais temas em pauta nas discussões médicas do período. A possibilidade desse tipo de terapia foi anunciada ao mundo ainda em meados dos anos 1940 pelos médicos Louis Godman e Albert Gilman, da Universidade de Yale - que ficaram curiosos com os efeitos do gás mostarda sobre o desenvolvimento das células brancas do sangue - e na década de 1950, testes clínicos confirmaram sua eficácia (OLSON, 2002). Somente na década de 1970 a possibilidade de uso dos quimioterápicos chega aos Anais Brasileiros de Ginecologia. Em artigo de José Vieira de Lima Filho, assistente do Serviço de Ginecologia do Hospital da Lagoa no Rio de Janeiro, a quimioterapia era colocada como alternativa final no tratamento do câncer de mama. Isto porque, cirurgia e radioterapia teriam 
efeitos na área afetada, cabendo à medicação química uma função de relevo no tratamento. Os quimioterápicos seriam ministrados como forma complementar ao tratamento e nos casos em que a paciente não pudesse ser beneficiada com cirurgia ou radioterapia. Além da quimioterapia, o artigo trazia considerações sobre outras associações medicamentosas utilizadas no tratamento dos cânceres ginecológicos (LIMA FILHO, 1970). A utilização da terapêutica, no entanto, deveria se dar de forma cautelosa, levando em consideração as individualidades e particularidades de cada caso. Nesse sentido, o autor argumentava que os quimioterápicos usados no combate ao câncer agiam tanto sobre a doença quanto sobre o próprio paciente, com a diminuição de sua resistência e favorecimento da quebra do equilíbrio defensivo gerado pelo organismo. Nesse sentido, criticava o uso precoce de quimioterápicos no tratamento do câncer, antes da devida utilização e avaliação dos recursos da cirurgia e da radioterapia (IDEM).

O exame clínico das mamas também era destacado já como rotina e ressaltada sua importância nos procedimentos ginecológicos. Nesse sentido, era salientada a importância do médico se antever a paciente, dominando as técnicas do exame clínico das mamas e da identificação de tumores para investigação (ROTSTEIN, 1970). A literatura destacava que um exame ginecológico não seria completo sem o exame dos seios. Partindo do princípio de que quanto menor o tumor maior as probabilidades de cura, era endossada a defesa do exame clínico das mamas em toda rotina ginecológica, como forma de diagnosticar a doença precocemente (MACHADO, 1970).

Em linhas gerais, era ratificada a importância do diagnóstico precoce do tumor e da correta indicação terapêutica como recursos imprescindíveis à melhora nos resultados do tratamento. Nesse sentido, o prognóstico do câncer de mama dependeria, além dos fatores intrínsecos relativos ao tumor e da resistência da paciente à agressão tumoral, da precisa indicação cirúrgica, nem tão "econômica" que seja tudo insuficiente para cura, nem tão extensa que possa acarretar riscos desnecessários à paciente (LIMA FILHO, 1970b).

Da terapêutica, a cirurgia ainda configurava como principal método, sendo "terapêutica primária" do câncer de mama, complementada pela radiologia na grande maioria dos casos (IDEM, p. 250). No âmbito cirúrgico, a mastectomia, como principal terapêutica empregada no tratamento do carcinoma da mama, foi, ao longo dos anos, sofrendo variações que acompanhavam o processo de configuração e definição da própria doença. No número especial do Jornal Brasileiro de Ginecologia, de 1970, o médico João Sampaio Góes Junior apresentou um relato da prática executada no Departamento de Obstetrícia e Ginecologia da Faculdade de Medicina da Universidade de São Paulo. O autor argumentava que inicialmente era utilizada a técnica clássica de Halsted, e com o correr do tempo, foram feitas pequenas alterações na prática cirúrgica, em relação às incisões.

O médico se posicionava contrário ao uso do bisturi elétrico, que fora defendido pelos médicos, principalmente do Serviço Nacional do Câncer nos anos 1940/1950, como destacado anteriormente no texto, argumentando que o bisturi poderia produzir queimaduras na pele, prejudicando o processo de cicatrização. $\mathrm{O}$ autor rebatia ainda a ideia de que o bisturi elétrico diminuiria a difusão de células neoplásticas. Defendia, assim, uma maior diversificação na perspectiva terapêutica, com base numa melhor avaliação propedêutica da progressão da doença, no sentido de individualizar os procedimentos terapêuticos, e de ser "radical apenas na medida exata" (GÓES JUNIOR, 1970: 251).

Somada à atenção ao trabalho do médico no exame sistemático das mamas a todo atendimento ginecológico, como elemento de detecção precoce da doença, era destacada ainda, nesse momento, a importância de um trabalho de educação e convencimento da população de que a detecção e tratamento precoces do carcinoma mamário eram os meios mais eficientes para 
obtenção dos maiores índices de cura da doença, ou da ampla sobrevida da paciente (IDEM). Num momento em que havia um maior consenso na definição e configuração da doença e de suas formas de tratamento, outras questões ganhavam maior espaço nas discussões médicas, ocupando a agenda de ações, como melhorias na sobrevida da paciente e campanhas de educação e prevenção.

\section{Considerações finais}

Ao tomar os Anais Brasileiros de Ginecologia como principal fonte na construção desse trabalho, destacamos o papel que publicações especializadas têm na configuração de pensamentos e práticas, moldando saberes, agendas e perfis profissionais. As percepções e definições sobre o câncer de mama construídas no periódico foram fruto de escolhas e disputas dos médicos e grupos envolvidos com a temática, e direcionaram os entendimentos e a construção das ações de controle da doença.

Na primeira metade do século XX, o câncer de mama seria um dos mais complexos problemas clínicos com os quais se depararia um ginecologista. Isso porque, mesmo sendo o de maior incidência, sua terapêutica ainda não apresentava desenvolvimento satisfatório. Os discursos publicados na revista representavam os conhecimentos e questões sobre a doença no Brasil, com dois momentos bem demarcados. Um primeiro, que correspondia ao início das preocupações médicas com a mesma, de sua construção como categoria médica, em termos de conceitualização, formas de detecção e possibilidades de tratamento. Nesse sentido, definir a doença e pensar no seu diagnóstico avançavam em sentidos comuns. O segundo momento, já com uma definição mais consensual em relação a alguns dos pontos debatidos até então, como categorização, tratamento, as discussões se apresentaram de forma mais pontual, trazendo um aprofundamento dos pontos já debatidos e levantando outros aspectos, como críticas à cirurgia radical e qualidade de sobrevida da paciente.

Em ambos os momentos, é possível perceber os esforços de constituição do campo de conhecimentos sobre o câncer de mama e da demarcação dos territórios de atuação dos médicos especialistas. Foi possível perceber a relação das questões debatidas pelos médicos brasileiros ao estado das discussões mais gerais sobre a doença, e da própria organização de um aparato institucional para o seu controle, incluindo desde mecanismos de prevenção, disgnóstico precoce, até tratamento e qualidade de vida da paciente. Para além dos artigos publicados, sendo os Anais Brasileiros de Ginecologia a principal revista em ginecologia no período, as discussões ali trabalhadas caminhavam no sentido de consolidação da própria ginecologia como especialidade médica.

\section{Referências}

ANAIS BRASILEIROS DE GINECOLOGIA - Editorial, vol, 1, n. 1, 1936.

ANAIS BRASILEIROS DE GINECOLOGIA - Editorial, vol. 7, n. 3, 1939.

ANAIS BRASILEIROS DE GINECOLOGIA - Editorial, vol 34, n. 1, 1952.

ARONOWITZ, Robert A. Do Not Delay: Brest Cancer and Time, 1900-1970. Milbank Quartely, New York, vol. 79, n. 3, p. 355-386, 2001.

BONAN, Claudia; TEIXEIRA, Luiz Antonio and NAKANO, Andreza Rodrigues. Absorção e metabolização dos hormônios sexuais e sua transformação em tecnologias contraceptivas: percursos do pensamento médico no Brasil. Ciência e saúde coletiva. Rio de Janeiro, vol.22, n.1, p.107-116, 
2017.

CANTOR, David. Cancer in the twentieth century. Baltimore: John Hopkins University Press, 2008.

CANTOR, David. Introduction: Cancer Control and Prevention in the Twentieth Century. Bulletin of the History of Medicine. Baltimore, volume 81, number 1, Spring 2007.

CONTI, S. Câncer do Seio. Tese apresentada à Faculdade de Medicina do Rio de Janeiro. Rio de Janeiro: 1912

COUTINHO, Alberto. Cancer da Mama. Anais Brasileiros de Ginecologia, Rio de Janeiro, Vol, 11, n. 2, p. 205-237, 1941.

COUTINHO, Alberto. Radiodiagnose mamária. Anais Brasileiros de Ginecologia, Rio de Janeiro, Vol. 22, n. 6, p. 453-466, 1946.

FERREIRA, Clarisse do Amaral. Professor Arnaldo de Moraes 1893-1961- in memorian. Anais Brasileiros de Ginecologia. vol, 51, n. 4, p. 211-215, 1961.

FREITAS, Patrícia de. A mulher é seu útero. A criação da moderna medicina feminina no Brasil. Antíteses, Londrina, vol. 1, n. 1, pp. 174-187, jan.- jun. de 2008.

GUIMARÃES, Ugo Pinheiro. Trauma e câncer mamário. Anais Brasileiros de Ginecologia, Rio de Janeiro, Vol. 14, n. 2, p. 325-338, 1942.

GÓES JR, João Sampaio. Mastectomia. Jornal Brasileiro de Ginecologia, Rio de Janeiro, vol. 72, n. 3, p. 209-220, 1970.

KROEFF, Mario. Resenha da luta contra o câncer no Brasil. Rio de Janeiro: Documentário do Serviço Nacional do Câncer, 2007.

LANA, Vanessa. Organização da especialidade médica e controle do câncer do colo do útero no Brasil: o Instituto de Ginecologia do Rio de Janeiro em meados do século XX. História, Ciências, Saúde - Manguinhos, Rio de Janeiro, v.23, n.3, p.683-701, jul.-set. 2016.

LANA, Vanessa. Ferramentas, práticas e saberes: a formação de uma rede institucional para a prevenção do câncer do colo do útero no Brasil, 1936-1970. 2012. Tese (Doutorado em História das Ciências e da Saúde) - Casa de Oswaldo Cruz, Fiocruz, Rio de Janeiro.

LEAVELL, Hugh; CLARK, Gurney. Medicina Preventiva. São Paulo: McGrew-Hill, 1976.

LIMA FILHO, José Vieira de. Considerações sobre o tratamento químico e hormonal do câncer genito-mamário feminino. Jornal Brasileiro de Ginecologia. Rio de Janeiro, Vol. 69, n. 1, p. 21-41, 1970.

LIMA FILHO, José Vieira de. Elementos da avaliação prognóstica do carcinoma mamário. Jornal Brasileiro de Ginecologia .Rio de Janeiro, vol. 72, n. 4, p. 249-266, 1970 (b).

LOWY, Ilana. Cancer, women and public health: the history of screening for cervical cancer. História, Ciências, Saúde - Manguinhos, Rio de Janeiro, vol. 17, supl. 1, julho 2010.

MACHADO, E. F. Santos. Importância do exame sistemático das mamas. Jornal Brasileiro de Ginecologia, Rio de Janeiro, vol. 72, n. 3, p. 177- 182, 1970.

MARTINS, Ana Paula Vosne. Visões do feminino: a medicina da mulher nos séculos XIX e XX. Rio de Janeiro: Editora FIOCRUZ, 2004.

MONTEIRO, Aurélio. Comedocarcinoma da mama. Anais Brasileiros de Ginecologia, Rio de Janeiro, Vol. 26, n. 6, 325-338, 1946.

MORAES, Arnaldo de. A propósito da terapêutica do carcinoma mamário - editorial. Anais Brasileiros de Ginecologia, Rio de Janeiro, Vol. 27, n. 2, p. 113-116, 1949.

MOSCUCCI, Ornella. The Science of Woman: Gynecology and Gender in England 1800 - 1829. Cambridge: Cambridge University Press, 1990. 
NUNES, Everardo Duarte. Análise de alguns modelos utilizados no ensino das ciências sociais nas escolas médicas. Revista de Saúde Pública. São Paulo, n.12, p. 506-515, 1974.

OLSON, James. Bathsheba's Breast: Women, Cancer and History. Baltimore: John Hopkins University Press, 2002.

OUDSHOORN N. Beyond the natural body: an archeology of sex hormones. London, New York: Routledge; 1994.

PORTO, Marco Antonio Teixeira; TEIXEIRA, Luiz Antonio e SILVA, Ronaldo Corrêa Ferreira da. Aspectos históricos do controle do câncer de mama no Brasil. Revista Brasileira de Cancerologia. Vol. 59, n. 3, p. 331 - 339, 2013.

ROSENBERG, Charles. The Tyranny of Diagnosis: Specific Entities and Individual Experience. IThe Milbank Quarterly. New York, Vol. 80, n. 2, p. 237 - 260, 2002.

ROSENBERG, Charles. Introduction: Framing disease: Illness, society and history. In: ROSENBERG, Charles e GOLDEN, Janet (editors). Framing Disease - Studies in Cultural History. New Brunswick, Rutgers University Press, 1997.

ROTSTEIN, Simão. Mama: o órgão esquecido do clínico. Jornal Brasileiro de Ginecologia. Vol. 72, n. 3, p. 175-176, 1970.

SANTOS JUNIOR, Dyonisio F. dos. Ligeiras considerações sobre o cancer de mama e o methodo Halsted. Tese da Faculdade de Medicina da Bahia. Salvador, 1923.

SILVEIRA, Anny Jackeline Torres e NASCIMENTO, Dilene Raimundo. A doença revelando a história. Uma historiografia das doenças. In: Nascimento, Dilene Raimundo e Carvalho, Diana Maul (orgs). Uma história brasileira das doenças. Brasília: Paralelo 15, 2004.

TEIXEIRA, Luiz Antonio; GRUZMAN, Carla. Os Materiais Educativos para a Prevenção do Câncer no Brasil: da perspectiva histórica à dimensão discursiva. In: Bertucci, Liane Maria; Mota, André; Schraiber, Lilia Blima. (Org.). Saúde e Educação: um encontro plural. 1ed. Rio de Janeiro: Editora Fiocruz, 2017.

TEIXEIRA, Luiz Antonio e ARAUHO NETTO, Luiz Alves. Still Controversial: Early detection and Screening for breast Cancer in Brazil, 1950 - 2010s. Medical History, v. 64, p. 52-70, 2020.

TEIXEIRA, Luiz Antonio e FONSECA, Cristina. De doença desconhecida a problema de saúde pública: o INCA e o controle do câncer no Brasil. Rio de Janeiro: Ministério da Saúde, 2007.

VIANNA, João. Amputação total da mama pela eletro-cirurgia. Anais Brasileiros de Ginecologia. Vol. 9, n. 5, p. 387-394, 1940.

VIVEIROS, Renato, ZANOTTA, C. A. e PINHO, Ana Lidya. Diagnóstico do Câncer da Mama. Conduta do Instituto de Ginecologia da Universidade do Brasil. Anais Brasileiros de Ginecologia. Vol. 41, n. 4, p. 201-208, 1956.

ZANOTTA, C. A. Valor do exame sistemático das mamas em ginecologia. Anais Brasileiros de Ginecologia, vol. 39, n. 2, p. 83-90, 1955.

Artigo recebido em: 02/10/2019

Artigo aceito em: 01/02/2020 